\title{
Optimizing of Real-time Multi-scale Display of DICOM Image based on CUDA
}

\author{
Yangping Wang \\ School of Mathematics and Statistics, \\ Lanzhou University, \\ Lanzhou, 730030, China \\ E-mail: w_yp73@163.com
}

\begin{abstract}
The multi-scale display of digital imaging and communications in Medicine (DICOM) is the important step of detecting the lesions and making the radiotherapy plan. It is achieved by the dynamic regulation of the multi-scale display which also is called window operation to observe the organ tissues with different densities. The traditional way utilizing the linear function fails to be consistent with the characteristics of the human eye and loses image details. In this paper, nonlinear multi-scale display method is utilized to extend the gray level of the region of interest. In order to solve the contradiction between the long computing time of the nonlinear function and the real time of the dynamic window operation, the parallel generation algorithm of the images is implemented on the CUDA platform. The experiment results have shown that the optimization method has reached more elaborate display and at least an order of magnitude faster than traditional algorithms.
\end{abstract}

Keywords—Multi-scale Display; CUDA; Medical Image

\section{INTRODUCTION}

Digital Imaging and Communications in Medicine (DICOM) ${ }^{[1]}$ has been developed into an international standard of image and relevant information transmission between devices in medical imaging informatics which simplifying the medical image information exchange and widely used in the actual medical ${ }^{[2]}$. In fact, the medical image produced by CT, MRI, PET, ultrasound, all conforms to the DICOM standard.

Obtaining image-related information and displaying medical image on other platforms is crucial. The medical image data is stored by the relative value whose gray-scale is generally 4096 or higher. In practice, its gray-scale is far higher than the maximum gray-scale of the monitor which is generally 256. So the medical image data needs to be mapped to the gray-scale according with the specification of the display device.

\author{
Yangping Wang, Peizhao Jiang, Yue Shi \\ School of Electronic and Information Engineering, \\ Lanzhou Jiaotong University, \\ Lanzhou, 730070, China \\ E-mail: yue_0667@163.com
}

At present, a majority of techniques about the DCIOM image display focus on the window operation of the multiscale display method by linear function and fast implementation ${ }^{[3,4]}$. A considerable number of techniques pay close attention to the intensification of the DICOM image after transformed ${ }^{[5,6]}$. However, the research about the window operation by nonlinear function to enhance the effect of the image display is lacking. Some researchers focus on introducing speediness look-up table to promote the speed which ensures the real-time. But the effect is unsatisfied. In this paper, the idle GPU computing power is exploited to complete the complex computing ${ }^{[7,8]}$.

In this paper the real-time window operation of the multi-scale display method by nonlinear function is implemented fleetly which makes the image appears more consistent with the human Contrast Sensitivity and highlights the ROI (Region of Interest). The parallel computing based on the CUDA of NVIDIA GPU assures the real-time ability.

\section{Proposed Method and Materials}

\section{A. Window Operation of Multi-scale Display Method}

The gray level of DICOM image is much larger than the monitor. However, there is a corresponding grayscale range for particular organs and tissues. For example, the range of CT value of the brain tissue is generally from $-100 \mathrm{Hu}$ to $150 \mathrm{Hu}$. So we can display grayscale range of interested organizations by compressing it onto the gray interval which the displayer can show and shield others. Adjusting the grayscale scope to observe the organ tissues and lesions in different densities is called window operation of the multiscale display method. The compressed grayscale range is window. The width of the range is the window width and its central value is window center. For CT images, the CT value in a certain range is transformed to the gray value of the displayer which is shown in Fig. 1. 


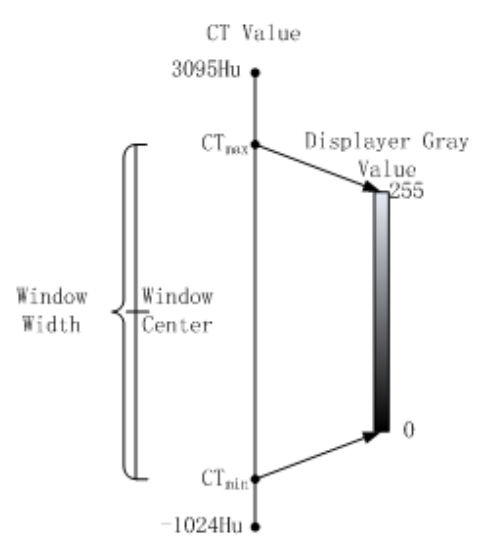

Fig. 1. Window Operation

$C T_{\min }$ is the minimum of the grayscale scope. $C T_{\max }$ is the maximum of the grayscale scope.

$$
\begin{aligned}
& \text { Window Width }=C T_{\max }-C T_{\min } \\
& \text { Window Center }=\left(C T_{\max }+C T_{\min }\right) / 2
\end{aligned}
$$

When Photometric Interpretation is MONOCHROME1 the mapping formula $K_{P}^{i}$ from the $M \times N \times K$ DICOM image $P$ to the $M \times N \times L$ DIB image $Q$ is as follow.

$$
K_{p}^{i}= \begin{cases}2^{L}-1 & i<C-\frac{W}{2} \\ g(i) & C-\frac{W}{2} \leq i \leq C+\frac{W}{2} \\ 0 & i>C+\frac{W}{2}\end{cases}
$$

$W$ is the window width. $C$ is the window center. $g(i)$ is the mapping from $K_{P}$ to $L_{Q}$ which satisfies formula (4).

$$
\forall i \forall j\left(i \in K_{P} \wedge j \in K_{P} \wedge i \leq j \rightarrow L_{Q}^{i} \geq L_{Q}^{j}\right)
$$

When Photometric Interpretation is MONOCHROME2 the mapping formula from the $M \times N \times K$ DICOM image $P$ to the $M \times N \times L$ DIB image $Q$ is as follow.

$$
K_{P}^{i}= \begin{cases}0 & i<C-\frac{W}{2} \\ f(i) & C-\frac{W}{2} \leq i \leq C+\frac{W}{2} \\ 2^{L}-1 & i>C+\frac{W}{2}\end{cases}
$$

$f(i)$ is the mapping from $K_{P}$ to $L_{Q}$ and satisfies the formula (6).

$$
\forall i \forall j\left(i \in K_{P} \wedge j \in K_{P} \wedge i \leq j \rightarrow L_{Q}^{i} \leq L_{Q}^{j}\right)
$$

The two picture color systems are related and inverted, namely, $g(i)=2^{L}-1-f(i)$. Therefore, in the paper, we only study the DICOM image whose color system is MONOCHROME2.

The window operation of the multi-scale display method can be sub-divided into linear window operation, piecewise linear window operation and nonlinear window operation according to the function types of $f(i)$.

\section{B. Nonlinear window operation}

Introducing nonlinear window operation is meaningful due to the disadvantages of linear window operation as follows: (1) the linear window operation cannot guarantee that the VOI occupies more gray levels in the generated DIB images and the nonlinear window operation can meet the preceding requirements while retaining the image information of other values and enhance image quality; (2) the grayscale of the DICOM image extremely high (e.g. the grayscale of Digital Radiography images is $2^{14}$ ). The detail will lost by linear window operation when the window is large; (3) human contrast sensitivity is distinctly nonlinear within the Luminance Range of the Grayscale Standard Display Function ${ }^{[9]}$; (4) people will produce different brightness sensation for the same luminance in the different brightness work environment according to Weber's law ${ }^{[10] .}$

This paper focuses on representative functions such as Sigmoid function, Logarithmic function, Exponential $f$ unction, Power function and so on.

a) The basic form of Sigmoid function is shown in Equation (7).

$$
\operatorname{Sigmoid}(x)=\frac{1}{1+e^{-x}}
$$

The domain of $\operatorname{Sigmoid}(x)$ is $(-\infty,+\infty)$. The range of $\operatorname{Sigmoid}(x)$ is $(0,1)$. It's a smooth continuous increasing function in domain which meets the requirement of formula (6). It is adjusted to Equation (8) when applied for nonlinear window operation.

$$
f(i)=\frac{255}{1+e^{-b \times \frac{i-C}{W}}}, i \in(-\infty,+\infty)
$$

Where $b$ relates to the gradient of the curve. For the bone window, the window center $C=300$ and the window width $W=1500$. The curve of function $f(i)$ is shown in Fig. 2 when $b=4$.

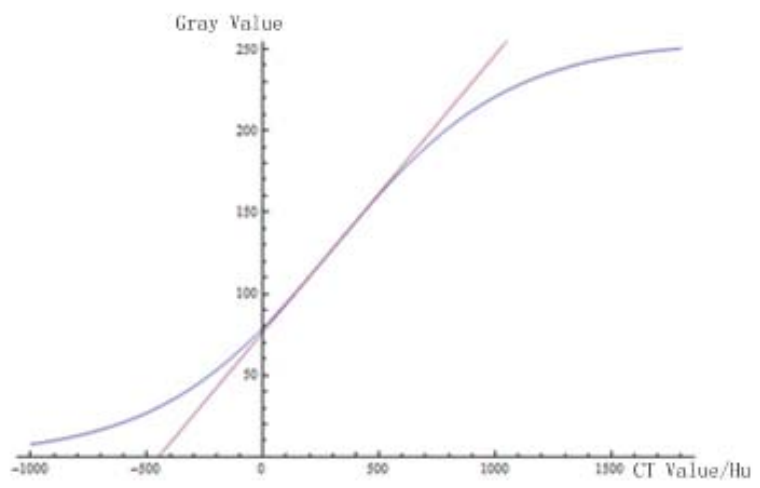

Fig. 2. The Curve of Sigmoid Function 
The intersection points of the tangent line at the window center point with $f(i)=0$ and $f(i)=255$ are the minimum of the window and the maximum of the window respectively.

$$
\begin{aligned}
& \text { Min }=C-\frac{W}{2}=-450 \\
& \operatorname{Max}=C+\frac{W}{2}=1050
\end{aligned}
$$

As can be seen from the shape of the curve, the values in the window occupy most gray region of the generated DIB image. Bring the Equation (9) and (10) back into Equation (8) could calculate the proportion $p$ of the number of the values in the window to all values.

$$
\begin{aligned}
p & =(f(\text { Max })-f(\text { Min })) / 255 \\
& =\frac{255}{1+e^{-b / 2}}-\frac{255}{1+e^{b / 2}}
\end{aligned}
$$

If $b=4, p=76.16 \%$. Namely, the Sigmoid function can exhibit the ROI particularly while retaining the image information of other region that offsets the deficiency of the linear window operation .

b) The basic form of Power function is shown in Equation 12.

$$
h(x)=c x^{r}+b
$$

The parameters $c$ and $r$ are positive numbers. If $r=1$ the power function turns to the linear function.

It is adjusted to Equation(13) when applied for nonlinear window operation.

$$
f(i)=255 \times\left(\frac{i-C+\frac{W}{2}}{W}\right)^{r}
$$

When the value of $r$ is between 0 and 1 this function could expand the lower gray values and compress the higher gray values so that the intensity distribution of the image is more consistent with human eye sensitivity just like the logarithmic function. When the value of $r$ is greater than 1 this function is converse just like the exponential function. The power function could take the place of the logarithmic and exponential function by adjusting parameter. Therefore, in this paper, we utilize the law function whose value of $r$ is generally less than 1 in the nonlinear window operation.

The power function is also used in gamma correction of the display device. Currently, almost all of the photoelectric conversion of the CRT monitor, the electronic camera and the photographic film are nonlinear.

For the lung window, the window center $C=-400$ and the window width $W=1500$. There are the curves of $r=0.2, r=0.4$ and $r=0.6$ from top to bottom shown in Figure 7.When $r=0.2$ the curve is too steep. When $r=0.6$ the curve is close to the linear function. Hence we select $r=0.4$.

\section{The Real-time Window Operate based on CUDA}

The nonlinear window operation of the multi-scale display method introduce transcendental function. The calculated amount is increased sharply that causes the delay of the image creation. The real-time window operation needs to ensure that the image is updated quickly and should not have the hysteresis phenomenon. The efficiency of image generation must be improved.

The calculate process of each pixel is independent. So the parallel computing method can be utilize to shorten the calculation time. The idle GPU computing power is exploited to complete the parallel computing. Nonlinear problems particularly benefit from the NVIDIA SFU (Special Function Units) that calculate several transcendental functions (such as $\log (), \exp (), \sin (), \cos ()$, and others) approximately 25 times faster than conventional processors $^{[11]}$.

CUDA is a parallel computing platform and programming model invented by NVIDIA which enables dramatic increases in computing performance by harnessing the power of the GPU ${ }^{12]}$. CDUA is a heterogeneous computing networks comprised by the CPU and GPU. The CPU is host and the GPU is device. The GPU stream multiprocessor is invoked by the CPU. CUDA enables programmers to program on the GPU and play its formidable power of parallel processing and floating point computing. The serial code runs on the host and the parallel code executes on the device.

Due to the fact that the calculate process of each pixel is independent, each pixel is assigned one thread. Since the different thread don't need synchronization, more blocks and threads are enabled to improve the speed.

It must be aware that conditionals (if statements) can greatly decrease performance inside an SM (Streaming Multiprocessor), as each branch of each conditional must be evaluated. There are a considerable number of conditionals in the piecewise linear window operation. However, it is not difficult for us to perceive that the Sigmoid function and line window operation are quite similar and the Sigmoid function is more smooth whose domain is $(-\infty,+\infty)$ and equation is constant at every point. That means the Sigmoid function doesn't need the conditional. Therefore, the Sigmoid function which is more excellent replaces the piecewise linear function.

In order to facilitate health care doctors to operate the window and view the image dynamically, the MFC (Microsoft Foundation Classes) of Microsoft Visual Studio 2010 is utilized to program the user interface and mouse messages which the program calls the kernel of CUDA. The suffix of CUDA files is ".cu" which could not include in the ".cpp" files directly. It requires the attended mode of extern "C" to call kernel function and setting the generated method of CUDA file, the entire project dependencies and generate event. 
By using the thread index, the block index, the block size and the grid size in the program to determine the location of the data in the input array directly can index the data easily, reduce the data transmission and complete the read in parallel correctly.

The main steps of program execution is shown as the following:

Step 1 Open the DICOM image file;

Step 2 Extract the information of relevant tags;

Step 3 Set Window Width and Window Center;

Step 4 Calls GPU kernel function and calculate DIB image pixel data according;

Step 5 Return the DIB pixel data array, fill the data structure of DIB and display the DIB image;

Step 6 If the Window Width and Window Center need be reset, return to Step 3, otherwise go to step 7 .

Step 7 End.

\section{EXPERIMENTS AND RESULTS}

The experiment environment is VS2010 based on C++. The computer we used for experiments consists of Intel Celeron E3300 @ 2.5GHz, NVIDIA 's GeForce GTX650 Ti, Microsoft Windows XP Professional Service Pack 3 and 2G of RAM.

For the DR image whose grayscale is higher, by compared the display effects of the window operation of multi-scale display method by the linear function shown in fig.3(a) and the Sigmoid function shown in fig.3(c), it was observed in the experiments that the image created by the Sigmoid function has the capability that not only shown the skeleton more clearly but also the edge of the body surface. It gives more detailed positional displacement information of the patient for the health care doctors.

For the CT image, by compared the display effects of the window operation by the linear function (Fig. 3(b)) and the power function (Fig.3(d)), it was observed in the experiments that the image created by the Power Function reveals more details of soft tissues whose gray value is in lower region and makes up for the weak ability of showing soft tissues of CT.

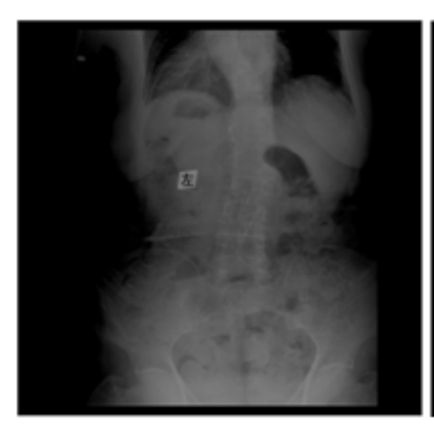

(a)

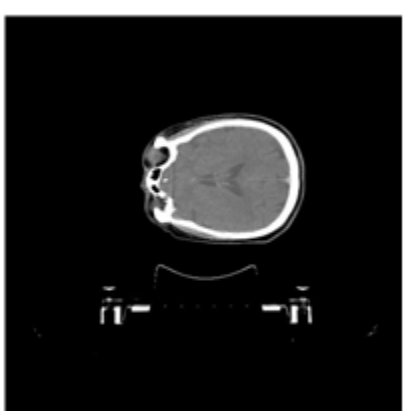

(b)

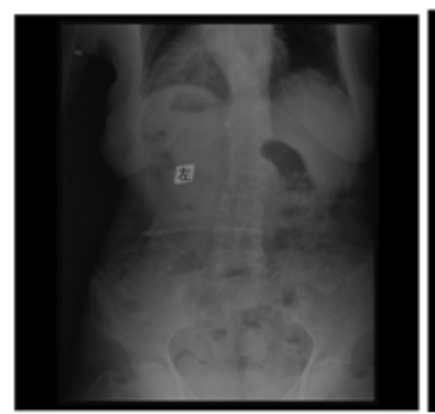

(c)

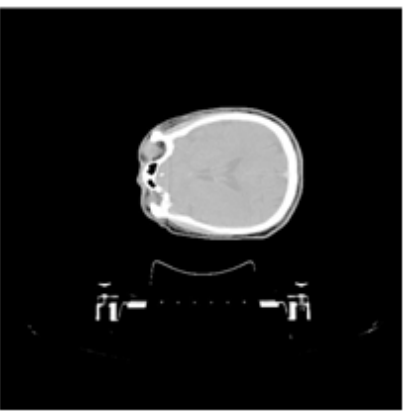

(d)
Fig.3 Experiment images and registration results. (a) and (b) are the window operates of DR image and CT respectively by the Linear Function. (c) the window operate by the Sigmoid Function. (d) the window operate by the Power Function.

It is apparent that utilizing the window operation by the nonlinear function has the capability of displaying the image excellently and lays the foundation for image segmentation.

We generate the CT image 5 times by different ways of the multi-scale display whose resolution is $512 * 512$ and record the elapsed time in milliseconds and speed-up ratio. The results are shown in Tab. 1.

Tab. 1. The Statistics Of CT Image

\begin{tabular}{cccc}
\hline & $\begin{array}{c}\text { CPU } \\
\text { time- } \\
\text { consuming }\end{array}$ & $\begin{array}{c}\text { GPU } \\
\text { time- } \\
\text { consuming }\end{array}$ & $\begin{array}{c}\text { Speed- } \\
\text { up } \\
\text { Ratio }\end{array}$ \\
\hline $\begin{array}{c}\text { The Linear } \\
\text { Function }\end{array}$ & 3.67 & 0.30 & 12.23 \\
$\begin{array}{c}\text { The Power } \\
\text { Function }\end{array}$ & 9.20 & 0.84 & 10.88 \\
$\begin{array}{c}\text { The Sigmoid } \\
\text { Function }\end{array}$ & 14.43 & 0.44 & 33.56 \\
\hline
\end{tabular}

We generate the DR image 5 times by different ways of the multi-scale display whose resolution is $3027 * 2560$ and record the elapsed time in milliseconds and speed-up ratio. The results are shown in Tab.2.

Consequently, utilizing GPU to generate the DIB pixel data array achieves a significant improvement of the computing speed which ensures the real-time of the dynamic window operation and provides the convenience for the study of medical images.

Tab. 2. The Statistics Of DR Image

\begin{tabular}{cccc}
\hline & $\begin{array}{c}\text { CPU } \\
\text { time- } \\
\text { consuming }\end{array}$ & $\begin{array}{c}\text { GPU } \\
\text { time- } \\
\text { consuming }\end{array}$ & $\begin{array}{c}\text { Speed- } \\
\text { up } \\
\text { Ratio }\end{array}$ \\
\hline The Linear Function & 92.66 & 3.00 & 30.77 \\
The Power Function & 609.50 & 23.75 & 25.66 \\
The Sigmoid Function & 412.50 & 8.04 & 51.29 \\
\hline
\end{tabular}




\section{CONCLUSION}

We have proposed window operation of the multi-scale display method by nonlinear function. In order to solve the contradiction between the long elapsed time which is taken for computing the nonlinear function and the real-time of the dynamic window operation, the parallel computing on GPU is introduced. In the study, the real-time multi-scale display is realized and the quality of the image is optimized. The maximum speed-up ratio can reach 51 times and the generated images has shown more details too. The next research work will focus on instruction optimization, improving the throughput of the instruction and the utilization of the memory bandwidth to further improve the speed of image generation.

\section{Acknowledgment}

This work is jointly supported by National Natural Science Foundation (60962004, 61162016), and Gansu International Science and Technology Cooperation Projects (144WCGA162).

\section{References}

[1] Vakente F., Viana-Ferreira C., Costa C. A Restful Gataway for Multiple Medical Image Repositories. Information Technology in Biomedicine. vol. 16, no. 3, pp. 356-364, 2012

[2] Vossberg M., Toldorff T., Krefting D. DICOM Image Communication in Globus-Based Medical Grids. Information Technology in Biomedicine. vol. 12, no. 2, pp. 145-153, 2008.

[3] Yang Y., Su Z., Sun L. Medical image enhancement algorithm based on wavelet transform. Electronics Letters. vol. 46, no. 2, pp. x-y, 2010.

[4] Rabbani H., Nezafat R., Gazor S. Wavelet-Domain Medical Image Denoising Using Bivariate Laplacian Mixture Model. Biomedical Engineering. vol. 56, no. 12, pp. 2826-2837, 2009.

[5] Panetta K., Agaian S. et al. Parameterized Logarithmic Framework for Image Enhancement. Systems, Man, and Cybernetics. vol. 41, no. 2, pp. 460-473, 2011.

[6] Tzu-Cheng Jen, Sheng-Jyh Wang. Bayesian Structure-Preserving Image Contrast Enhancement and its Simplification. Circuits and Systems for Video Technology. vol. 22, no. 6, pp. 831-843, 2012.

[7] Qi Zhang, Eagleson R., Peters T.M. GPU-Based Visualization and Synchronization of 4-D Cardiac MR and Ultrasound Images. Information Technology in Biomedicine. vol. 16, no. 25, pp. 8578890, 2012.

[8] Broxvall M., Emilsson K., Thunberg P. Fast GPU Based Adaptive Filtering of 4D Echocardiography. Medical Imaging. vol. 31, no. 6, pp. 1165-1172, 2012.

[9] Shi Junsheng, Yao Juncai, Yu Hongfei et al. Measurement of luminance contrast sensitivity function of human vision system on cathode ray tube display. Acta Optica Sinica.vol. 24, no. 4, pp. 744748, 2007.

[10] Winkler S. Issues modeling for perceptual video quality assessment. Signal Processing. vol. 78, no. 2, pp. 231-252, 1999.

[11] Young-Jun Kim, Hyo-Eun Kim, Seok-Hoon Kim. Homogeneous Stream Processors With Embedded Special Function Units for HighUtilization Programmable Shaders. Very Large Scale Integration (VLSI) Systems. vol. 20, no. 9, pp. 1691-1704, 2012

[12] Xiang Su, Jiaji $\mathrm{Wu}$ et al. GPU-Accelerated Computation for Electromagnetic Scattering of a Double-Layer Vegetation Model.
Selected Topics in Applied Earth Observations and Remote Sensing. vol. 6, no. 4, pp. 1799-1806, 2013 